\title{
Cardiovascular magnetic resonance imaging in assessment of intracaval and intracardiac extension of renal cell carcinoma
}

\author{
Vimal Raj, FRCR, ${ }^{\text {a,b }}$ Francisco Alpendurada, MD, ${ }^{\mathrm{b}}$ Timothy Christmas, FRCS, ${ }^{\mathrm{d}}$ Neil E. Moat, FRCS, ${ }^{\mathrm{c}}$ and \\ Raad H. Mohiaddin, FRCR, FRCP ${ }^{b, e}$
}

\begin{abstract}
Objectives: About 1 in 5 patients with renal cell carcinoma have intravascular tumoral extension at presentation. Level of tumoral extension within inferior vena cava determines surgical approach, with higher extension requiring cardiopulmonary bypass. Tumoral invasion of inferior vena caval wall is associated with poor prognosis. We evaluated accuracy of magnetic resonance imaging (MRI) in assessing level of intravascular extension of renal cell carcinoma and predicting vessel wall invasion.
\end{abstract}

\begin{abstract}
Methods: MRIs and surgical database were reviewed from January 1999 to December 2008. Sixty-four patients with suspected intravascular extension of renal cell carcinoma underwent MRI. Forty-one underwent curative or palliative surgery at our institution and were included in final analysis. MRI scans were reviewed to determine intravascular extension and tumoral adherence to the vessel wall, as assessed by circumferential flow around the intravascular tumor and its mobility during different phases of cardiac cycle. MRI findings were correlated with surgical findings to assess accuracy.
\end{abstract}

Results: There was $87.8 \%$ agreement $(P<.001 ; \kappa=0.82)$ between MRI and surgical findings regarding level of intravascular extension of tumor. MRI was highly sensitive and specific (93\%) in assessing supradiaphragmatic extension (negative predictive value, 96\%). Depending on sign used, sensitivities and negative predictive values in assessing tumoral adherence to vessel wall ranged from $86 \%$ to $95 \%$ and $81 \%$ to $91 \%$, respectively.

Conclusions: MRI is highly accurate in staging intravascular and intracardiac extension, aiding in accurate preoperative surgical planning. MRI may help determine prognosis of renal cell carcinoma by accurately assessing tumoral adherence to the vessel wall. (J Thorac Cardiovasc Surg 2012;144:845-51)

Renal cell carcinoma (RCC) accounts for 3\% to $4 \%$ of cancers in the United States, ${ }^{1}$ and its worldwide incidence and mortality are increasing at $2 \%$ to $3 \%$ per decade. ${ }^{2}$ At presentation, venous extension is reported to occur in $20 \%$ of patients. The inferior vena cava (IVC) is involved in as many as $15 \%$ of all patients with RCC, and right atrial involvement is seen in approximately $1 \%$ of cases. ${ }^{3-6}$

Surgical management is preferred, because RCC is uniformly resistant to chemotherapy and not sensitive enough to radiotherapy. ${ }^{7-9}$ Intravascular tumoral extension is not associated with an adverse prognosis, provided

\footnotetext{
From the Department of Radiology, ${ }^{\text {a }}$ Glenfield Hospital, University Hospitals of Leicester NHS Trust, Leicester, United Kingdom; the Cardiovascular Magnetic Resonance Unit ${ }^{\mathrm{b}}$ and the Department of Cardiac Surgery, ${ }^{\mathrm{c}}$ Royal Brompton and Harefield Foundation Trust, London, United Kingdom; the Department of Surgery, ${ }^{\mathrm{d}}$ Royal Marsden Hospital, London, United Kingdom; and the National Heart and Lung Institute, ${ }^{\mathrm{e}}$ Imperial College London, London, United Kingdom.

Supported by the NIHR Cardiovascular Biomedical Research Unit of Royal Brompton and Harefield NHS Foundation Trust and Imperial College of London.

Disclosures: Authors have nothing to disclose with regard to commercial support.

Received for publication July 26, 2011; revisions received Nov 1, 2011; accepted for publication Nov 18, 2011; available ahead of print Dec 19, 2011

Address for reprints: Raad H. Mohiaddin, FRCR, FRCP, Cardiovascular Magnetic Resonance Unit, Royal Brompton and Harefield Foundation Trust, Sydney St, London SW3 6NP, UK (E-mail: R.Mohiaddin@ rbht.nhs.uk).

$0022-5223 / \$ 36.00$

Copyright (C) 2012 by The American Association for Thoracic Surgery doi:10.1016/j.jtcvs.2011.11.035
}

a complete resection is possible. ${ }^{6,10}$ Extension into the IVC, however, is associated with a poorer survival outcome than is extension into the renal vein. ${ }^{11}$ The level of tumoral extension within the IVC determines the surgical approach used, with supradiaphragmatic extension requiring cardiopulmonary bypass (CPB) with or without deep hypothermic circulatory arrest. ${ }^{12,13}$ It is thus vital to determine the level of intravascular tumoral extension preoperatively. This not only facilitates surgical planning but also allows appropriate involvement of cardiac surgical teams.

Significant difference in prognosis exists between patients with tumoral invasion of the IVC wall and those with free-floating tumor in the IVC. ${ }^{14,15}$ Hatcher and colleagues ${ }^{14,16}$ reported the 5 -year survivals in the 2 groups to be $25 \%$ and $69 \%$, respectively. Direct tumoral invasion of IVC wall, into surrounding soft tissue, can be determined by multidetector computed tomography (MDCT) or magnetic resonance imaging (MRI; Figure 1). ${ }^{17,18}$ Recognition of vessel wall invasion at early stages, when tumor is adherent to the vessel wall, can be difficult to assess by any imaging modality.

In this study, we looked at the accuracy of cardiovascular MRI in assessing the level of intravascular tumoral extension of RCC and the ability of MRI to predict vessel wall invasion. 


\section{Abbreviations and Acronyms \\ $\mathrm{CPB}=$ cardiopulmonary bypass \\ IVC = inferior vena cava \\ MDCT $=$ multidetector computed tomography \\ MRI = magnetic resonance imaging \\ $\mathrm{NPV}=$ negative predictive value \\ $\mathrm{RCC}=$ renal cell carcinoma}

\section{MATERIALS AND METHODS \\ Patients}

Cardiovascular MRIs and the surgical database of a single tertiary cardiothoracic referral center (Royal Brompton and Harefield Foundation Trust) were reviewed between January 1999 and December 2008. All patients who underwent MRI for assessment of known RCC with suspected vascular extension were included in the study. Demographic data, intraoperative findings, and pathology reports were collated. Patients who underwent curative or palliative surgery at our institution were included in the final analysis. Patient consent was not deemed to be necessary by the local research and ethics committee.

\section{MRI Scan Protocols}

During the period of study, all the scans were performed in 1 of our 3 1.5-T systems (2 Avanto and 1 Sonata; Siemens Medical Systems, Erlangen, Germany). Standard scan protocol included (Table 1) the following: half-Fourier acquisition single-shot turbo spin echo and cinematic steady-state free precession sequences of the heart in standard cardiac planes (2-, 3-, and 4-chamber and short-axis views) to establish cardiac anatomy and function. Half-Fourier acquisition single-shot turbo spin echo and single-shot steady-state free precession images of the abdomen and pelvis (axial and coronal planes) were acquired to assess anatomy and extent of local and vascular tumoral invasion. A stack of cinematic steady-state free precession images covering the IVC was acquired in 2 planes to assess tumoral invasion of the vessel wall. Gadoliniumenhanced renal angiography in arterial and venous phases was used to detect level of vascular invasion and wall invasion. Toward the latter half of the study, volumetric interpolated breath-hold examination sequence of the abdomen was performed after angiography (Figure 2).

\section{MRI Data Analysis}

MRI images were reviewed, independent of the surgical findings, by 2 authors (V.R. and F.A.) with 5 or more years of experience in cardiovascular MRI. Consensus opinion was used for statistical analysis. Specific analysis was made regarding the level of intravascular tumoral extension by stratifying patients into 4 groups according to the level of intravascular extension per the classification proposed by Neves and Zincke ${ }^{19}$ (Figure 3): involvement of the renal vein (level I), involvement of the infrahepatic IVC (level II), involvement of the intrahepatic IVC (level III), and supradiaphragmatic extension (level IV). To assess tumoral adherence to the vessel wall, 2 specific MRI signs were analyzed. First sign looked at circumferential blood flow around the intravascular tumor component on true fast imaging with steady-state precession and postcontrast images (Figure 4). Second sign looked at the mobility of the intravascular extension on cinematic images during different phases of the cardiac cycle. Tumor was considered to be adherent to the vessel wall if there was no circumferential flow around it, if it was not mobile within the vessel, or both.

\section{Statistical Analysis}

Statistical analysis was performed with Stata software (version 10; StataCorp LP, College Station, Tex). MRI staging of intravascular extension

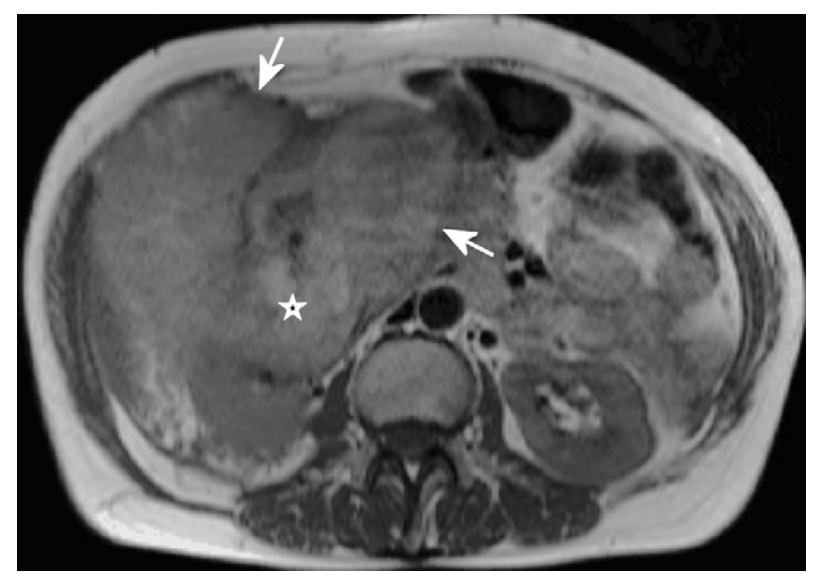

FIGURE 1. Half-Fourier acquisition single-shot turbo spin echo axial image of upper abdomen showing right renal cell carcinoma (star) with extensive peritoneal soft tissue extension (arrows).

was compared with intraoperative staging to assess agreement by means of $\kappa$ statistics. The accuracy of MRI in predicting the level of intravascular extension was calculated by assessing sensitivity and specificity values for individual stages, with the surgical findings considered the criterion standard. The MRI findings of circumferential flow and tumoral mobility were specifically analyzed against the intraoperative finding of tumoral adherence to the vessel wall. The sensitivity and specificity of MRI in predicting the need for CPB (levels III and IV) were also assessed.

\section{RESULTS \\ Study Population}

A total of 64 patients with RCC underwent MRI as part of the staging process during the period of study. There were 34 men and 30 women with a median age of 62 years, (range, 24-83 years). Forty-three patients had right kidney tumors, 19 had left-sided tumors, and 2 patients had bilateral RCC. MRI scan was generally well tolerated by patients, with only a couple of incidents in which suboptimal images were obtained because patients were unable to breath hold. On average, the scan duration was less than an hour, and delayed myocardial enhancement imaging after gadolinium contrast was performed in patients with known or suspected coronary artery disease.

\section{Surgical Management}

Of the 64 patients, 10 were not suitable candidates for radical or palliative surgery in view of their comorbidities or metastatic spread of the disease. These patients were referred to the oncologists or a palliative care team and were excluded from this analysis. Twelve patients were referred back to their primary hospitals for further management and were excluded from the analysis because operative details were not available. One patient underwent exploratory laparotomy and was excluded from this analysis. In total, 41 patients who underwent curative or palliative surgery at our institution were included for statistical analysis. Average time lag between the MRI 
TABLE 1. Magnetic resonance imaging protocol

\begin{tabular}{|c|c|c|c|c|c|}
\hline & HASTE & SSFP SS & SSFP cine & CE-MRA & VIBE \\
\hline TR/TE & $700 / 42$ & $292.1 / 1.22$ & $40.2 / 1.13$ & $2.85 / 1.19$ & $5.73 / 2.64$ \\
\hline Flip angle & $160^{\circ}$ & $80^{\circ}$ & $80^{\circ}$ & $15^{\circ}$ & $10^{\circ}$ \\
\hline \multicolumn{6}{|l|}{ Field of view } \\
\hline Read (mm) & 340 & 340 & 320 & 350 & 350 \\
\hline Phase $(\%)$ & 75 & 81.3 & 68.8 & 75 & 75 \\
\hline \multicolumn{6}{|l|}{ Acquisition } \\
\hline Base resolution & 256 & 256 & 192 & 384 & 256 \\
\hline Phase resolution $(\%)$ & 59 & 65 & 100 & 80 & 70 \\
\hline Slice resolution $(\%)$ & - & - & - & 79 & 64 \\
\hline Slices per slab & - & - & - & 96 & 64 \\
\hline Slice thickness (mm) & 6 & 6 & 7 & 1.3 & 2.5 \\
\hline Phases per cardiac cycle & - & - & 25 & - & - \\
\hline Bandwidth (Hz/pixel) & 781 & 888 & 930 & 690 & 250 \\
\hline iPAT & GRAPPA $\times 2$ & GRAPPA $\times 2$ & - & GRAPPA $\times 2$ & GRAPPA $\times 2$ \\
\hline
\end{tabular}

HASTE, Half-Fourier acquisition single-shot turbo spin echo; SSFP, steady-state free precession; SS, single shot; cine, cinematic; $C E-M R A$, contrast-enhanced magnetic resonance angiogram; VIBE, volumetric interpolated breath-hold examination; TR, repetition time; TE, echo time; $i P A T$, integrated parallel acquisition techniques; GRAPPA, generalized autocalibrating partially parallel acquisition.

scan and the surgery was 30 days (range, 1-91 days). Time lag was less than 30 days for 25 patients and more than 60 days for 4 patients. Three patients, 2 undergoing palliative surgery and 1 with level II extension, did not require $\mathrm{CPB}$.

\section{MRI Versus Surgical Findings}

The MRI and surgically determined levels of intravascular tumoral thrombus extension are compared in Table 2. There was $87.8 \%(P<.001 ; \kappa=0.82)$ agreement between MRI and intraoperative findings in assessing the level of

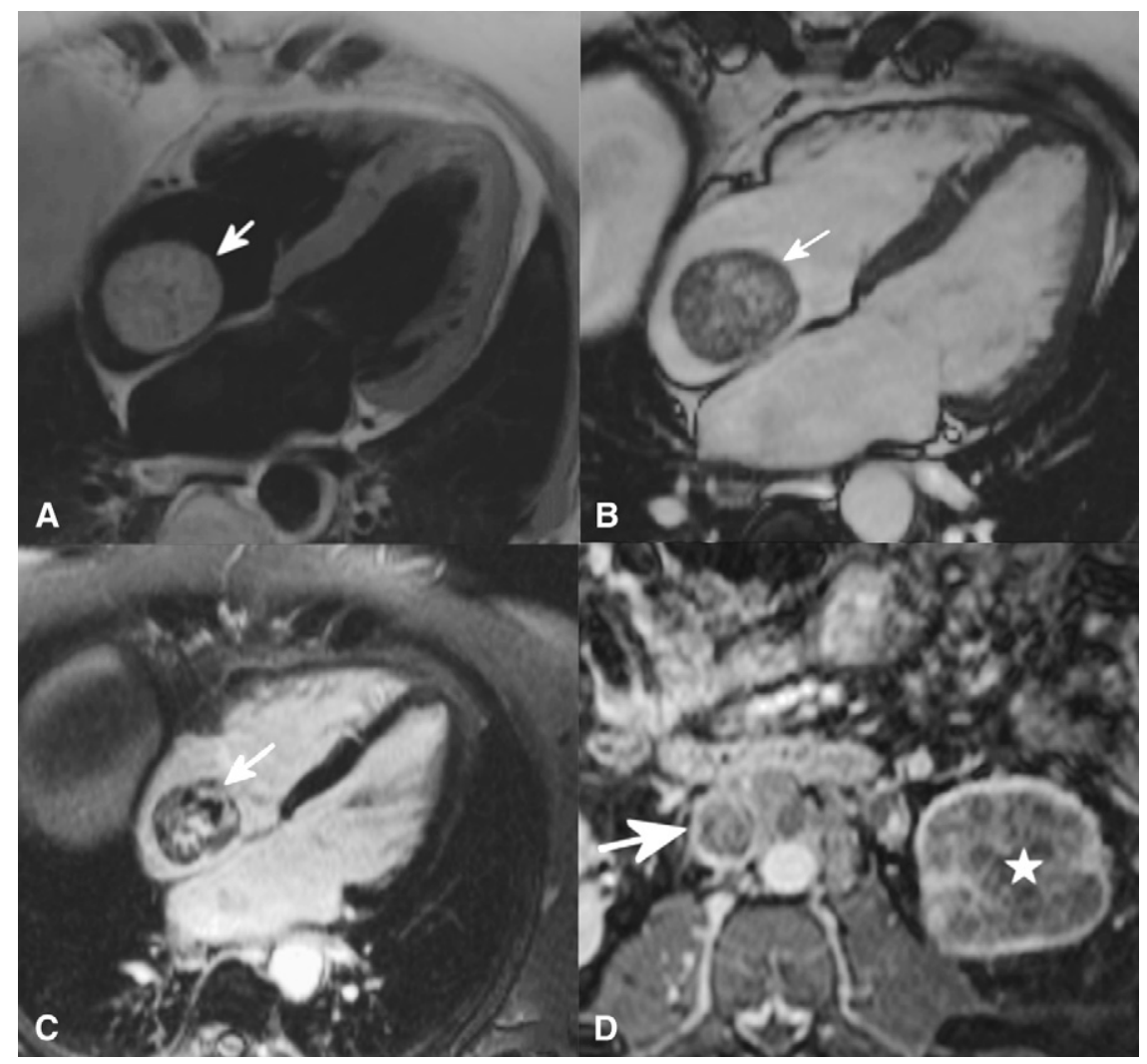

FIGURE 2. Different sequences in axial plane used for assessing a patient with left renal cell carcinoma with suspected intravascular extension. A, HalfFourier acquisition single-shot turbo spin echo; B, steady-state free precession; C, postcontrast. Images show the right atrial extension of tumor (arrow). D, Volumetric interpolated breath-hold examination image shows left renal cell carcinoma (star) with inferior vena caval tumoral thrombus (arrow). 


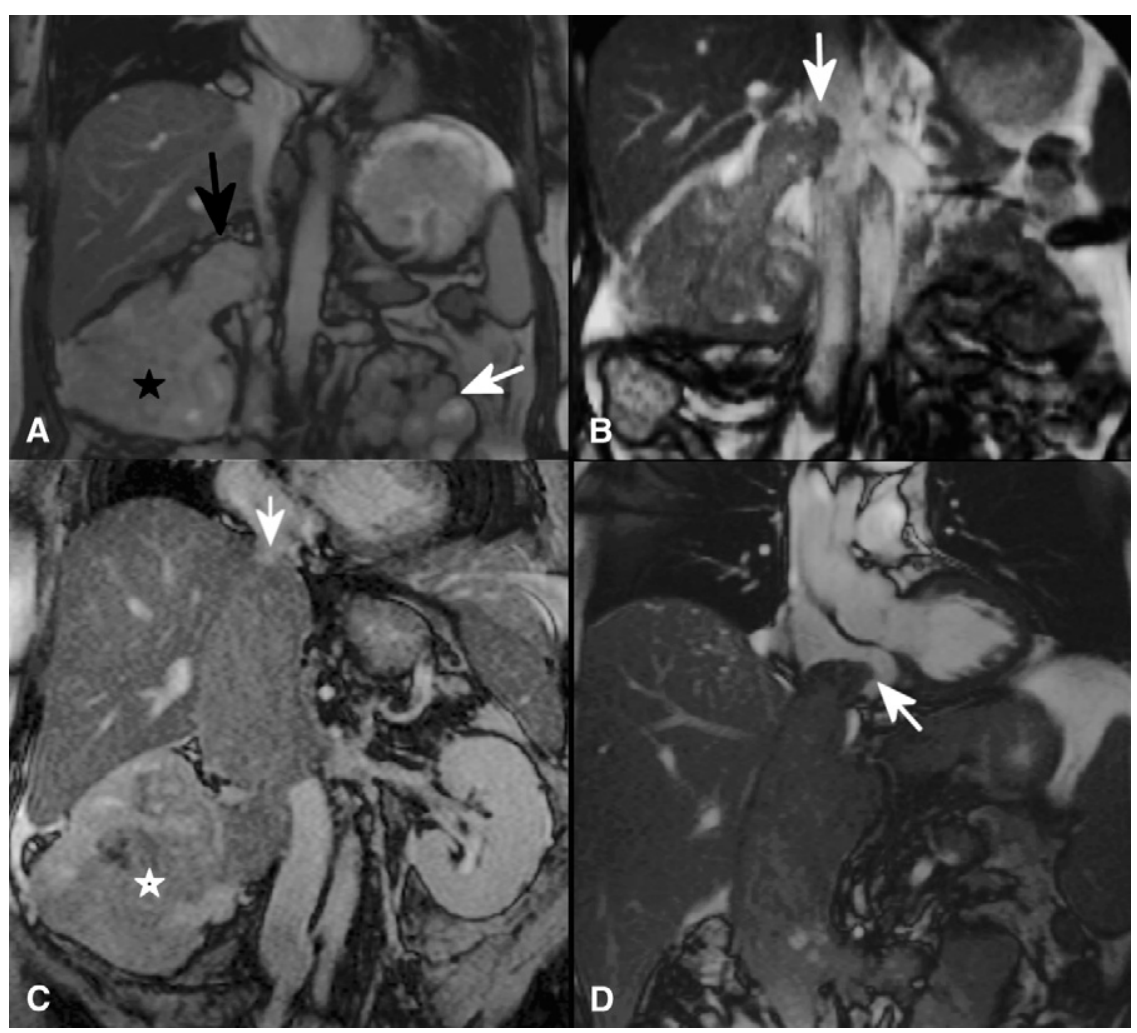

FIGURE 3. Steady-state free precession static images in coronal plane depicting different levels of intravascular tumoral extension. A, Level I, renal vein extension (black arrow) of right renal cell carcinoma (star). Also note left polycystic kidney (white arrow). B, Level II, infrahepatic inferior vena caval extension (arrow) of right renal cell carcinoma. C, Level III, hepatic inferior vena caval extension (arrow) of the right renal cell carcinoma (star). D, Level IV, supradiaphragmatic extension (arrow) into the right atrium.

intravascular extension of tumor. The sensitivities, specificities, and negative predictive values (NPVs) of MRI in determining different levels of intravascular extension are shown in Table 3. MRI was best in predicting level IV extension, with sensitivity and specificity of $93 \%$ and NPV of $96 \%$. Specific analysis was made of levels III and IV together, because both these levels necessitate cardiac surgical team involvement during nephrectomy. All patients with level IV extension on MRI required right atrial excision for tumor removal, apart from 1 patient who underwent palliative surgery for the abdominal disease. The sensitivities, specificities, and NPVs of the 2 MRI signs in assessing tumoral adhesion to the vessel wall are shown in Table 4 . Both signs were accurate in predicting tumoral adherence to the IVC wall $(P<.001)$. Mobility of intravascular extension on MRI had a higher NPV (92\%) in assessing tumoral adherence to the vessel wall.

Of the 12 patients referred back to the primary hospital, 1 had no vascular extension, 2 had level I extension, and 9 had tumor extending into infrahepatic IVC (level II). Four patients who had medical management had distant metastases (1 level 0, 2 level II, and 1 level IV), 2 were not amenable to surgery (1 level II and 1 level IV), and 4 were not thought to be medically fit to undergo surgery ( 3 level II and 1 level IV).

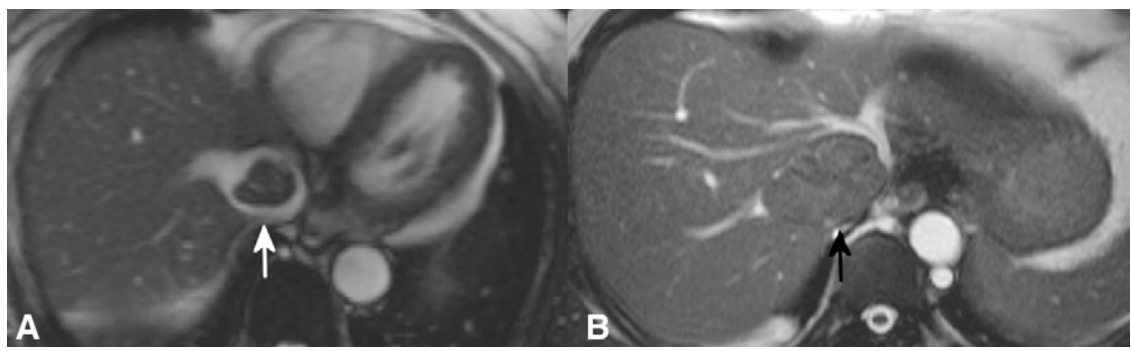

FIGURE 4. Axial steady-state free precession images of 2 patients with level IV disease. Note circumferential flow around tumoral extension on the left (A, white arrow) and no flow around the lesion on right (B, black arrow). 
TABLE 2. Level of intravascular tumoral thrombus extension on magnetic resonance imaging versus level determined at surgery

\begin{tabular}{llrcr}
\hline & \multicolumn{4}{c}{ Surgery } \\
\cline { 2 - 5 } MRI & I & II & III & IV \\
\hline I & 1 & 0 & 0 & 0 \\
II & 0 & 13 & 1 & 0 \\
III & 0 & 1 & 9 & 1 \\
IV & 0 & 0 & 2 & 13 \\
\hline
\end{tabular}

MRI, Magnetic resonance imaging.

\section{Incidental Findings}

Two of our patients were incidentally found to have cardiac disease (left ventricular aneurysm and aortic stenosis). The patient with aortic stenosis underwent a simple nephrectomy and is under close surgical review for potential aortic valve replacement. Ventricular aneurysm in the other patient was secondary to previous myocardial infarction, and this patient had undergone coronary artery bypass grafting. Nonsurgical management was deemed appropriate after discussion with the patient. MRI also demonstrated soft tissue, visceral, and bony metastases in 15 patients, precluding them from curative surgery. Seven patients had thrombus in the pulmonary arteries, and 1 had a segmental liver infarct as a result of tumoral extension into the IVC (Figure 5).

\section{DISCUSSION}

Surgical resection is the only effective treatment for RCC. ${ }^{20,21}$ Accurate preoperative staging of RCC is vital, because the surgical approach to tumor resection varies according to the level of intravascular tumoral extension. Different imaging modalities, including MDCT, MRI, and transesophageal echocardiography, are currently used to determine intravascular tumoral extension. There is limited published evidence regarding the accuracy of these imaging modalities relative to intraoperative findings. To the best of our knowledge, this is the largest single-center study looking at the accuracy of cardiovascular MRI in assessing the intravascular tumoral extension and its role in providing prognostic information in patients with RCC.

There was significant agreement between the MRIdetermined vascular extension and the intraoperative findings $(87.8 \% ; P<.001)$. The sensitivities, specificities, and NPVs of MRI were different for different levels of

TABLE 3. Sensitivities, specificities, and negative predictive values of magnetic resonance imaging for various levels of extension

\begin{tabular}{lccc}
\hline Predicted level of extension & Sensitivity & Specificity & NPV \\
\hline IV & $93 \%$ & $93 \%$ & $96 \%$ \\
III & $75 \%$ & $93 \%$ & $90 \%$ \\
$>$ II & $96 \%$ & $93 \%$ & $93 \%$ \\
\hline
\end{tabular}

$N P V$, Negative predictive value.
TABLE 4. Sensitivities, specificities, and negative predictive values of different magnetic resonance imaging observations in assessing tumoral adherence to the vessel wall

\begin{tabular}{lccc}
\hline Observation & Sensitivity & Specificity & NPV \\
\hline $\begin{array}{l}\text { Circumferential flow around } \\
\text { intravascular extension }\end{array}$ & $86 \%$ & $65 \%$ & $81 \%$ \\
$\begin{array}{l}\text { Mobility of intravascular } \\
\text { extension }\end{array}$ & $95 \%$ & $55 \%$ & $91 \%$ \\
\hline$N P V$, Negative predictive value. & & &
\end{tabular}

extension, with level IV assessment by MRI being the best (NPV, 96\%; sensitivity and specificity, 93\%). Level III and IV extensions usually warrant the involvement of cardiac surgical teams, because CPB with or without deep hypothermic cardiac arrest is required for adequate resection and hemostasis. MRI was highly sensitive $(96 \%)$ and specific $(93 \%)$ and had a good NPV $(93 \%)$ in predicting level III or IV extension, thereby indicating the need of CPB. Findings of our study are superior to previously reported MRI sensitivities of $80 \%$ to $85 \% .^{22,23}$

MRI was inaccurate in staging intravascular extension in 5 patients. One patient had understaging of the disease as level II (surgical level III) and 1 as level III (surgical level IV). No specific reason for this understaging is apparent. One plausible cause is rapid tumor growth between imaging and surgery; however, the time lags between imaging and surgery were not significant (27 and 33 days). Two patients had overstaging of disease as level IV (time lags, 7 and 8 days) and 1 as level III (time lag, 89 days). In all 5 cases, complete surgical excision was possible and did not require any significant change in operative management.

The presence and extent of tumoral thrombus in the IVC have limited influence on the prognosis, even when the thrombus extends to the right atrium; however, significant difference in prognosis exists between patients with tumoral invasion of the IVC wall and those with free-floating tumoral thrombus in the IVC. ${ }^{14-16}$ Assessment of IVC wall invasion by tumoral thrombus is thus an important part of any staging investigation. Although MDCT is the most widely used imaging technique for diagnosis and staging of RCC, it can be unreliable in assessing direct invasion of IVC wall by tumoral thrombus. ${ }^{4}$ The 2 MRI signs analyzed in this study were able to predict tumoral adherence to the IVC wall accurately $(P<.001)$. This information not only will help in better surgical planning but also will allow improved prognostication before surgery.

In our routine practice, patients with suspected RCC undergo MDCT examination at the outset. Cardiac MRI is performed in patients with suspected level II or greater vascular extension. Patients with suspected level III or IV extension are also assessed perioperatively with transesophageal echocardiography to ascertain supradiaphragmatic extension of tumor. The operative team includes an urologist, a cardiothoracic surgeon, and an anesthesiologist. In this 


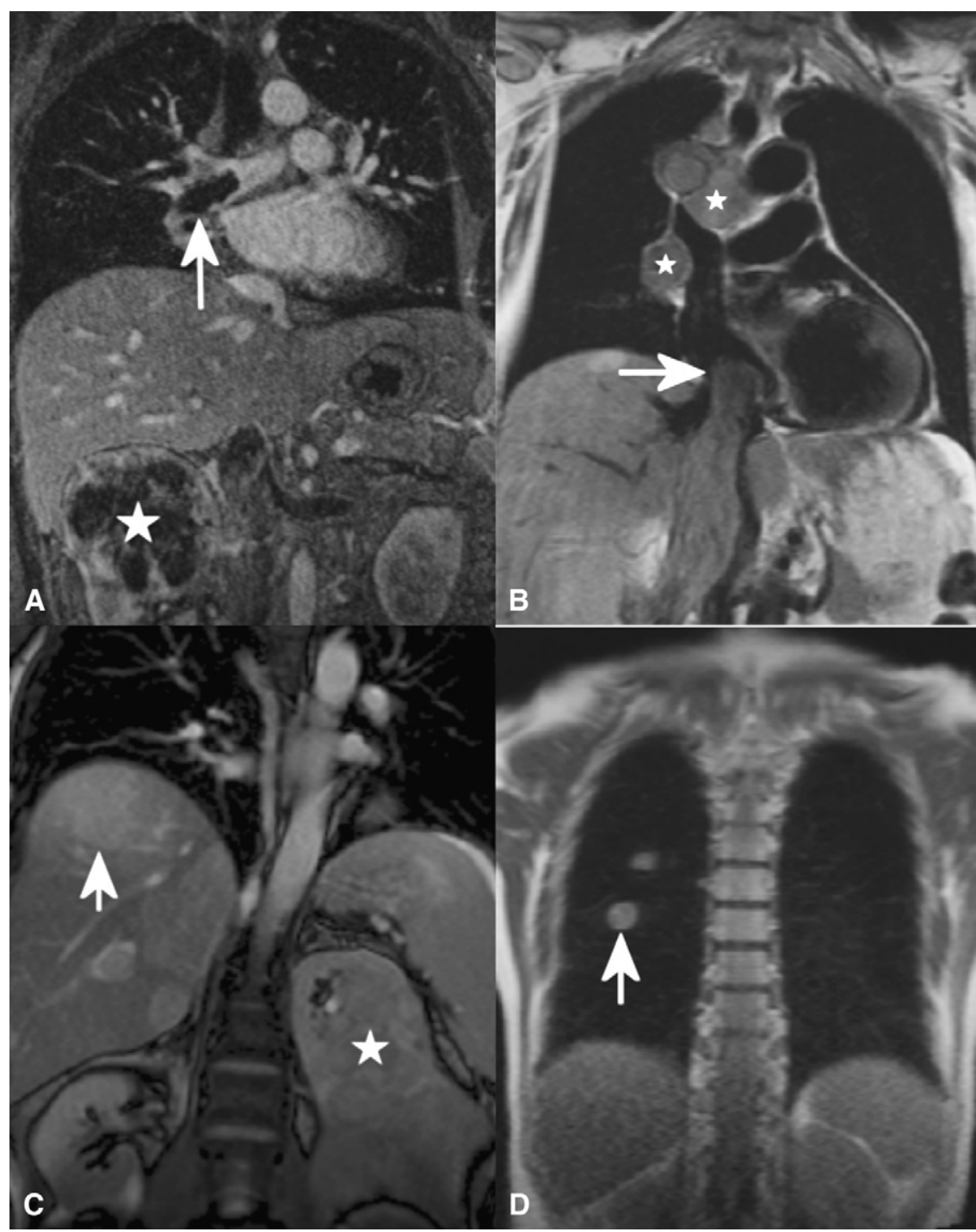

FIGURE 5. Coronal images showing extracardiac pathologies detected on cardiac magnetic resonance imaging. A, Thrombus in right pulmonary artery (arrow). B, Level IV tumor (arrow) with mediastinal lymph nodes (star). C, Liver infarct (arrow) secondary to left renal cell carcinoma (star). D, Multiple lung metastases (arrow).

clinical setting, cardiovascular MRI has a further advantage of assessing the cardiac functional status, especially for those people who will be undergoing CPB.

We do not suggest the use of cardiovascular MRI as a routine imaging examination for staging RCC. Instead, it should be used as an adjuvant modality in patients who have suspected or known vascular invasion and are under consideration for surgery. The main reason for this is the limited availability of technology and radiologic expertise in the field of cardiovascular MRI. On the other hand, MDCT is widely available and remains the ideal method for assessing distant metastases. ${ }^{3}$ Assessment of supradiaphragmatic and right atrial extension on MDCT, however, may require venous phase imaging with electrocardiographic gating. This will require cardiothoracic expertise and appropriate MDCT equipment, which may not be universally available.

We do recognize the limitations of our study. Similar to other studies on this subject, ${ }^{10}$ our study subjects comprised a highly selected patient population, with most of the patients having previously diagnosed or suspected intravascular extension and being referred for surgical assessment. This also accounts for a high incidence of CPB in our group. Some of the patients with level I or II disease also required CPB for excision of pulmonary tumoral emboli and bulky IVC involvement. The retrospective nature of the study may have led, as a result of the heterogeneity in imaging techniques, parameters, and coil types, to underestimation of the true performance of MRI. Images and reports from other modalities were not routinely available; performance 
of MRI and its ability to assess tumoral vessel wall adherence therefore could not be compared with MDCT or echocardiography. The true prognostic value of cardiac MRI-predicted tumoral adhesion and early invasion of the vessel wall was not specifically assessed in this study. The study was aimed at ascertaining the performance of MRI, and therefore no mortality or morbidity data were collected. Because of the lack of a control group (patients with RCC not undergoing MRI), it is not possible to assess the influence of MRI on the outcomes of these patients.

In summary, accurate preoperative staging of RCC is vital as surgical approach, and involvement of the cardiac surgical team will vary depending on the tumoral extension. MRI is highly accurate in staging intravascular and intracardiac extension. MRI may help in determining the prognosis of patients with RCC by accurately assessing tumoral adherence to the vessel wall.

\section{References}

1. American Cancer Society. Cancer facts and figures 2008. Atlanta: The Society; 2008.

2. Gupta K, Miller JD, Li JZ, Russell MW, Charbonneau C. Epidemiologic and socioeconomic burden of metastatic renal cell carcinoma (mRCC): a literature review. Cancer Treat Rev. 2008;34:193-205.

3. Kearney GP, Waters WB, Klein LA, Richie JP, Gittes RF. Results of inferior vena cava resection for renal cell carcinoma. J Urol. 1981;125:769-73.

4. Reznek RH. CT/MRI in staging renal cell carcinoma. Cancer Imaging. 2004;4 Spec No A:S25-32.

5. Rini BI, Campbell SC, Escudier B. Renal cell carcinoma. Lancet. 2009;373: 1119-32.

6. Schimmer C, Hillig F, Riedmiller H, Elert O. Surgical treatment of renal cell carcinoma with intravascular extension. Interact Cardiovasc Thorac Surg. 2004;3: 395-7.

7. Rini BI. New approaches in advanced renal cell carcinoma. Urol Oncol. 2005;23: 65-6.

8. Rouvière $\mathrm{O}$, Brunereau L, Lyonnet $\mathrm{D}$, Rouleau P. [Staging and follow-up of renal cell carcinoma]. J Radiol. 2002;83(6 Pt 2):805-24. French.
9. Mueller-Lisse UG, Mueller-Lisse UL. Imaging of advanced renal cell carcinoma. World J Urol. 2010;28:253-61.

10. Chiappini B, Savini C, Marinelli G, Suarez SM, Di Eusanio M, Fiorani V, et al. Cavoatrial tumor thrombus: single-stage surgical approach with profound hypothermia and circulatory arrest, including a review of the literature. $J$ Thorac Cardiovasc Surg. 2002;124:684-8.

11. Wagner B, Patard JJ, Méjean A, Bensalah K, Verhoest G, Zigeuner R, et al. Prognostic value of renal vein and inferior vena cava involvement in renal cell carcinoma. Eur Urol. 2009;55:452-9.

12. Montie JE, Pontes JE, Novick AC, Vanderburg Mendendorp S, Streem SB Montague DK, et al. Resection of inferior vena cava tumor thrombi from renal cell carcinoma. Am Surg. 1991;57:56-61.

13. Novick AC, Kaye MC, Cosgrove DM, Angermeier K, Pontes JE, Montie JE, et al Experience with cardiopulmonary bypass and deep hypothermic circulatory arrest in the management of retroperitoneal tumors with large vena caval thrombi. Ann Surg. 1990;212:472-7.

14. Ergen FB, Hussain HK, Caoili EM, Korobkin M, Carlos RC, Weadock WJ, et al MRI for preoperative staging of renal cell carcinoma using the 1997 TNM classification: comparison with surgical and pathologic staging. AJR Am J Roentgenol. 2004;182:217-25.

15. Russo P. Renal cell carcinoma: presentation, staging, and surgical treatment Semin Oncol. 2000;27:160-76.

16. Hatcher PA, Anderson EE, Paulson DF, Carson CC, Robertson JE. Surgical management and prognosis of renal cell carcinoma invading the vena cava. J Urol. 1991;145:20-4.

17. Cuevas C, Raske M, Bush WH, Takayama T, Maki JH, Kolokythas O, et al. Imaging primary and secondary tumor thrombus of the inferior vena cava: multidetector computed tomography and magnetic resonance imaging. Curr Probl Diagn Radiol. 2006;35:90-101.

18. Stern Padovan R, Perkov D, Smiljanic R, Oberman B, Potocki K. Venous spread of renal cell carcinoma: MDCT. Abdom Imaging. 2007;32:530-7.

19. Neves RJ, Zincke H. Surgical treatment of renal cancer with vena cava extension. Br J Urol. 1987;59:390-5.

20. Figlin RA. Renal cell carcinoma: management of advanced disease. J Urol. 1999; 161:381-7.

21. Motzer RJ, Russo P. Systemic therapy for renal cell carcinoma. J Urol. 2000;163 408-17.

22. Laissy JP, Menegazzo D, Debray MP, Toublanc M, Ravery V, Dumont E, et al Renal carcinoma: diagnosis of venous invasion with Gd-enhanced MR venography. Eur Radiol. 2000;10:1138-43.

23. Roubidoux MA, Dunnick NR, Sostman HD, Leder RA. Renal carcinoma: detection of venous extension with gradient-echo MR imaging. Radiology. 1992;182: 269-72. 